Documentation et bibliothèques

DOCUMENTATION BIBLIOTHËQUES

\title{
La formation des utilisateurs à la consultation des catalogues en ligne : ARCHIMEDE, BADADUQ et MUSE
}

\section{Hélène Bussière}

Volume 38, numéro 4, octobre-décembre 1992

URI : https://id.erudit.org/iderudit/1028769ar

DOI : https://doi.org/10.7202/1028769ar

Aller au sommaire du numéro

\section{Éditeur(s)}

Association pour l'avancement des sciences et des techniques de la documentation (ASTED)

\section{ISSN}

0315-2340 (imprimé)

2291-8949 (numérique)

Découvrir la revue

\section{Citer cet article}

Bussière, H. (1992). La formation des utilisateurs à la consultation des catalogues en ligne : ARCHIMEDE, BADADUQ et MUSE. Documentation et bibliothèques, 38(4), 203-206. https://doi.org/10.7202/1028769ar

Tous droits réservés (C) Association pour l'avancement des sciences et des techniques de la documentation (ASTED), 1992
Ce document est protégé par la loi sur le droit d'auteur. L'utilisation des services d'Érudit (y compris la reproduction) est assujettie à sa politique d'utilisation que vous pouvez consulter en ligne.

https://apropos.erudit.org/fr/usagers/politique-dutilisation/ 


\title{
La formation des utilisateurs à la consultation des catalogues en ligne : ARCHIMEDE, BADADUQ et MUSE *
}

\author{
Hélène Bussière \\ Bibliothèque centrale \\ Université du Québec à Montréal
}

Les catalogues interactifs ou catalogues en ligne (online public access catalogues ou OPAC) connaissent une grande popularité auprès des usagers. Ces systèmes demeurent toutefois complexes et obligent les utilisateurs à surmonter plusieurs difficultés dont les plus évidentes tiennent à la mémorisation du système par exemple les commandes, les points d'accès, la syntaxe pour la formulation des demandes, les méthodes ou les techniques d'interrogation, etc. De plus, à ces difficultés s'ajoute celle de la question de recherche que l'usager doit formuler avant d'interroger le catalogue en ligne. Face à de tels problèmes, on peut se demander si une formation peut aider les usagers à surmonter les difficultés qui se présentent et, si oui, quelle incitation serait la plus appropriée ? Question qui, on s'en doute, n'est pas facile à résoudre, d'autant plus que ces activités de formation, lorsqu'elles sont offertes, provoquent souvent, chez le personnel, une certaine controverse : tâches répétitives dont on peut remettre en question l'utilité. Quant aux usagers eux-mêmes, beaucoup avouent ne pas avoir de temps à y consacrer. II en résulte que ces systèmes coûteux sont sousexploités et n'atteignent pas leur but principal qui est d'offrir un meilleur accès aux collections.

Nous avons visité trois bibliothèques scientifiques de Montréal mettant à la disposition de leurs usagers les catalogues en ligne suivants : ARCHIMEDE à la Bibliothèque de l'École Polytechnique, BADADUQ à la Bibliothèque des sciences de l'Université du Québec à Montréal (UQAM) et enfin MUSE, système utilisé à la Physical Sciences \& Engineering Library de l'Université McGill. Notre but est de comparer les programmes de formation dispensés aux usagers et de commenter ces observations à la lumière d'autres études.

À partir d'un questionnaire servant de canevas aux rencontres, nous avons interviewé trois bibliothécaires, soit une par site visité1. Ces bibliothécaires travaillent au sein des services de référence de leur bibliothèque. Elles sont toutes trois engagées dans le processus de la formation documentaire de leur service respectif. Nous commenterons les réponses obtenues auprès de ces trois bibliothécaires ${ }^{2}$.

\section{Modes d'apprentissage}

Nous avons tout d'abord demandé de quelle façon les usagers apprenaient, en général, à utiliser le catalogue en ligne.

L'auto-apprentissage semble être, chez les usagers des trois bibliothèques, la forme privilégiée d'apprentissage face aux OPAC. En effet, une majorité d'entre eux apprendraient à utiliser les OPAC, seuls ou entre amis. A McGill, on estime à $25 \%$ le nombre d'usagers qui apprennent par eux-mêmes ou entre amis. Ce pourcentage est cependant inférieur aux autres bibliothèques puisqu'on nous a répondu, à l'École Polytechnique, que c'était de cette façon que les usagers apprenaient le plus à utiliser ARCHIMEDE, tandis qu'à I'UQAM, on nous a dit que beaucoup d'utilisateurs recouraient à cette méthode. À la Bibliothèque de l'École Polytechnique, les usagers qui proviennent de disciplines scientifiques n'ont pas ou ont moins peur d'un terminal que les usagers provenant d'autres disciplines. De plus, les systèmes offrant des menus donnent à l'usager une fausse impression de facilité. Mais d'autres raisons expli- quent ce phénomène. Une enquête réalisée par le Council Library Resources (CLR) en $1981-1983^{3}$ révèle que lorsqu'on a demandé aux usagers pourquoi ils n'utilisaient pas le catalogue en ligne, la réaction la plus courante était qu'ils n'avaient pas le temps d'apprendre. Plus récemment, les résultats de l'étude de Cherry et Clinton à l'Université de Toronto ${ }^{4}$ vont dans le même sens que nos observations: $43,2 \%$ des usagers ont d'abord appris à utiliser FELIX par tâtonnements (trial and error).

* NDLR: Voir aussi l'article signé par Lisette Dupont: «La place de la formation documentaire dans la formation universitaire», publié dans le numéro de janvier-mars 1992 de Documentation et bibliothèques, p. 35-40. Le texte de cette présente chronique nous a été proposé avant la publication de l'article de Mme Dupont. Cette chronique fut à l'origine une recherche soumise dans le cadre d'un cours «Recherche en repérage et diffusion de l'information» donné à l'École de bibliothéconomie et des sciences de l'information de l'Université de Montréal, à l'hiver 1991.

1. Nous tenons à remercier ces bibliothécaires qui ont bien voulu nous accorder quelques minutes de leur temps précieux.

2. Dans la discussion qui suit, nous avons regroupé certaines questions de façon à faciliter la présentation des résultats.

3. J.R. Matthews et al., Using Online Catalogs : A Nationwide Survey, New York, Neal-Schuman, 1983, cité par Christine Borgman, "Why are Online Catalogs Hard to Use? Lessons Learned from Information-Retrieval Studies", Journal of the American Society for Information, vol. 37, no. 6 (1986), 396.

4. Joan Cherry and Marshall Clinton, «A Profile of User Background and User Satisfaction with the University of Toronto OPAC and the Implications for User Training and User Interfaces", Proceedings of the ASIS Annual Meeting, vol. 26, (1989), p. 123. 
On peut toutefois soulever la question suivante : est-ce que cette méthode est efficace? Autrement dit, est-ce que les usagers, en apprenant par eux-mêmes, utilisent ou exploitent au mieux les possibilités qu'offrent les systèmes des catalogues en ligne? Lorsqu'on demande aux bibliothécaires si les usagers surestiment leurs capacités face à l'utilisation des $O P A C$, elles ont toutes répondu sans hésiter qu'effectivement les usagers croient pouvoir utiliser les systèmes sans problèmes, ou encore qu'ils le connaissent bien ou sinon, que leur utilisation sera facile. Ce qui, à la lumière des commentaires que nous avons recueillis, ne semble pas le cas. À ce propos, il faut mentionner que les usagers de ces trois bibliothèques savent utiliser la logique booléenne dans un contexte scolaire, mais ne savent pas l'utiliser dans un contexte documentaire. Or, commele souligne Christine Borgman ${ }^{5}$, les programmes de formation (ainsi que les concepteurs de systèmes) devraient tenir compte de la méthode autodidacte. Toujours selon $\mathrm{C}$. Borgman, les systèmes devraient être conçus pour encourager l'auto-apprentissage (more self instructionnal).

Quant à la documentation imprimée, elle est très peu consultée par les étudiants des trois bibliothèques. Ces derniers préfèrent demander conseil plutôt que de consulter les guides ou dépliants mis à leur disposition. À McGill toutefois, on ne met pas de documentation imprimée à la disposition des usagers. Cette politique a été mise en place de manière à obliger les utilisateurs à demander l'aide aux bibliothécaires. Ces observations vont à l'encontre des résultats obtenus par l'Office of Research of the OCLC Online Computer Library Center ${ }^{6}$ et par Joan Cherry et Marshall Clinton ${ }^{7}$. En effet, ces études font ressortir l'importance du matériel imprimé auprès des usagers dans l'utilisation des OPAC. Comment expliquer cette divergence de vues ? Soulignons d'emblée que nos observations n'ont pas le caractère scientifique des résultats des études que nous venons de citer. Cependant, il suffit parfois de peu de choses pour faire toute la différence: la présentation matérielle d'un guide, par exemple, peut suffire à elle seule à encourager sa consultation. En ce qui concerne la politique de McGill, nous signalons le problème suivant : lorsqu'un usager se trouve à un étage supérieur, descendra-t-il au premier pour demander l'aide du bibliothécaire quand on sait que même à proximité des usagers, les bibliothécaires ne sont pas consultés pour diverses raisons: gêne, peur de poser une question futile ou ridicule, etc.

Karen Markey ${ }^{8}$ fait remarquer les avantages que présente le matériel imprimé : un dépliant, par exemple, peut rejoindre énormément d'usagers. Comparativement au personnel qui donne des séances de formation, le matériel imprimé constitue une méthode de formation peu coûteuse et «flexible». En effet, l'usagerva chercher la réponse au problème qui se pose et ce, chaque fois que le problème se présente.

En ce qui concerne, les séances de formation, nous avons obtenu une grande disparité dans les réponses. À l'École Polytechnique, les étudiants suivent très rarement des sessions de formation encadrées par le personnel. En fait, les séances de formation mises sur pied n'ont pas de succès auprès des usagers; très peu d'étudiants se présentent de telle sorte, qu'elles finissent toujours par être annulées. On explique ainsi ce phénomène: «De façon générale, les étudiants de premier cycle empruntent peu de documents, ils viennent à la bibliothèque pour $y$ travailler». La formation documentaire à la Polytechnique n'existe pour ainsi dire qu'au deuxième cycle et seulement à la demande des professeurs (environ 20 professeurs font une demande sur un nombre potentiel de 200). Au moment de notre rencontre, des séances de formation de quinze minutes étaient offertes à l'heure du dîner. Les bibliothécaires gardent cependant un certain espoir avec ce qu'ils appellent la formation individuelle. Lorsqu'un étudiant se présente au comptoir de référence, le bibliothécaire en profite alors pour expliquer à l'étudiant comment utiliser ARCHIMEDE : ces formations sont cependant de très courte durée et le contenu de celles-ci se limite au problème que pose le système ARCHIMEDE 9 . 950 étudiants (nouvellement arrivés) ont fait une demande d'aide pour le catalogue en ligne auprès des bibliothécaires de référence sur un potentiel de 2000 étudiants.

Les étudiants de la Bibliothèque des sciences de l'UQAM suivent eux aussi très rarement une formation encadrée par le personnel. Seuls les professeurs du département de biologie font une demande auprès du service de référence. Des séances de formation sont donc offertes aux étudiants rattachés à ce département pendant les deux premières semaines du trimestre d'automne. Ces séances ont une durée maximale de 45 minutes (pour $B A D A D U Q$ ) et une vingtaine d'étudiants au maximum peuvent y participer à la fois.

À McGill, à la bibliothèque scientifique, des séances de formation sont offertes pendant toutel'année scolaire. Cinq à sept personnes peuvent y participer au maximum et pendant une durée de 30 à 45 minutes. Des statistiques internes révèlent que $75 \%$ des étudiants suivent une session de formation. On estime par ailleurs que $40 \%$ de la tâche du bibliothécaire au comptoir de référence est consacrée à aider les usagers au moment où ils interrogent MUSE. Notons que c'est le seul endroit où les bibliothécaires se déplacent lorsque le système d'alarme du catalogue retentit ${ }^{10}$.

Cette disparité va s'expliquer un peu plus loin avec les réponses obtenues à d'autres questions; retenons pour le moment qu'un programme de formation ne va pas de soi. Plusieurs difficultés se dressent en effet : la motivation des étudiants, la participation des professeurs, le marketing des programmes, les ressources matérielles et humaines en sont quelques exemples.

Examinons de plus près le contenu des séances de formation offertes aux bibliothèques scientifiques de I'UQAM et

5. Christine Borgman, "Why are Online...», 396

6. Karen Markey, «Offline and Online User Assistance for Online Catalogue Searchers", Online (May 1984), 60-61.

7. Joan Cherry and Marshall Clinton, «A Profile of User...", p. 125.

8. Karen Markey, «Offline and Online...», 60-61.

9. Ce système n'offre pas d'accès au sujet de telle sorte que les étudiants utilisent souvent l'accès au titre en croyant à tort utiliser l'accès au sujet.

10. ARCHIMEDE n'est cependant pas muni d'un tel système. 
de McGill. Les nombreuses possibilités qu'offre BADADUQ obligent le personnel à aborder plusieurs éléments ou facettes du système : les différents accès, les commandes, la logique booléenne (et, ou, sauf) et les clés d'accès, plus particulièrement l'indexation qui, de façon générale, est formulée au masculin singulier. Le simple fait de regrouper vingt étudiants autour d'un seul terminal provoque la première difficulté : les étudiants voient mal les détails, perdent l'attention et l'intérêt nécessaire pour mémoriser un contenu aussi chargé.

Les séances de formation de la bibliothèque scientifique de McGill n'ont pas un contenu aussi dense car le système ne permet pas encore l'utilisation de la logique booléenne. Soulignons cependant que cette bibliothèque offre à ces usagers trois niveaux de formation qui correspondent aux cheminements scolaires des étudiants, lesquels entraînent des besoins documentaires différents selon qu'ils sont étudiants au niveau du baccalauréat, de la maîtrise ou du doctorat. Au premier niveau de formation, on explique aux sept étudiants regroupés autour d'un terminal ce que c'est qu'un catalogue et ce qu'il permet: les accès auteur, titre et vedettesmatière. Un aperçu des différentes commandes fait aussi partie de ce premier niveau de formation. Quant aux diverses possibilités du système, elles seront décrites tout au cours des trois niveaux de formation. Les deux derniers niveaux répondent aux besoins plus spécifiques des étudiants de maitrise et de doctorat.

De façon générale, toutes ces formations s'en tiennent à l'essentiel car on est conscient, dans les différentes bibliothèques, du temps limité d'attention que les utilisateurs sont prêts à accorder. De plus, tous ces usagers, regroupés autour d'un terminal, ont un rôle passif, ce qui ne les avantage guère dans la démarche d'apprentissage et de mémorisation. Ce phénomène dit de novices perpétuels résulte du fait que les usagers n'arrivent pas à mémoriser les différents éléments abordés lors de formations, si bien que l'apprentissage est toujours à recommencer. Notons ici que le matériel imprimé peut combler cette lacune. Quant à l'aide en ligne, elle n'est pour ainsi dire jamais utilisée car, en fait, elle ne joue pas le véritable rôle «d'aide», selon les bibliothé- caires consultées. II n'est pas étonnant, dès lors, que les usagers aillent plutôt demander conseil auprès d'amis ou encore demander l'aide aux bibliothécaires.

Nous avons aussi posé quelques questions concernant les microfichiers. $\grave{A}$ l'ÉcolePolytechnique, ces catalogues sont consultés très rarement. Àla Bibliothèque des sciences de l'UQAM, les usagers utilisent les microfichiers lorsque le catalogue en ligne ne leur permet pas de trouver ce qu'ils cherchent. De plus, il arrive que les étudiants ne consultent que les bibliographies données en cours et aillent ensuite sur les rayons afin de pratiquer le "browsing» qui, pourtant, pourrait très bien se faire à partir du catalogue en ligne. Ces derniers, avec la possibilité de la logique booléenne, n'ont-ils pas été conçus pour faciliter le «browsing» (balayage) à partir d'un terminal ? Ce phénomène montre à quel point les programmes de formation des usagers sont essentiels et aussi, quelle attitude entraîne une formation inadéquate: un manque de confiance dans les catalogues en ligne et, par conséquent, une sous-utilisation de systèmes qui, rappelons-le, sont extrêmement coûteux.

La bibliothèque scientifique de McGill ne met aucun microfichier à la disposition des usagers. Cette politique s'inscrit dans le même sens que pour le matériel imprimé qui, lui non plus, n'est pas disponible. De plus, l'absence des microfichiers permet d'indiquer aux usagers que la bibliothèque entre dans une nouvelle ère, et qu'un microfichier n'est plus adapté à ce nouveau contexte, comparativement bien sûr à la puissance qu'offre un catalogue en ligne. Nous considérons, quant à nous, que cette politique encourage concrètement l'adoption du catalogue en ligne. Ceci ne vaut toutefois qu'à la condition d'avoir un solide programme de formation afin de soutenir les utilisateurs dans leur démarche d'apprentissage et d'adaptation.

Dans les paragraphes quisuivent, nous nous pencherons sur la participation des professeurs, la mise en marché de la formation documentaire et la tenue de statistiques sur l'utilisation de catalogues ordinolingues.

\section{Participation des professeurs}

Les professeurs de l'École Polytechnique ne participent d'aucune façon à la formation documentaire saufau deuxième cycle et seulement dans certains cas. Cet accueil mitigé de la part des professeurs envers la formation documentaire s'expliquerait ainsi : ils considèrent que leurs cours sont déjà chargés et que le temps consacré à chaque cours suffit à peine à couvrir la mátière. C'est pourquoi ils n'alloueraient aucune période à la formation documentaire.

Rappelons qu'à la Bibliothèque des sciences de I'UQAM, seuls les professeurs du département de biologie font appel au service de référence afin d'offrir, entre autres, une initiation à BADADUQ.

À la Physical Sciences \& Engineering Library de l'Université McGill, les professeurs participent et encouragent la formation documentaire.

\section{Politique de marketing}

Àl'École Polytechnique, on commente ainsi cette politique: «Nous avons essayé toutes sortes de moyens, mais les étudiants ne se présentent pas ou, tout au plus, seulement quelques étudiants y assistent. Ces temps-ci, nous annonçons les sessions de formation par intercom. Nous allons devoir prendre une décision sous peu (à savoir si nous poursuivons) car très peu d'étudiants se présentent».

À la Bibliothèque des sciences de I'UQAM, voici la réponse obtenue: «Non, nous n'avons pas de politique de marketing pour la formation des usagers, et nous "gardons le profil bas» parce que nous n'avons pas les ressources financières et humaines nécessaires pour soutenir une demande qui serait plus élevée qu'en ce moment».

Le personnel de la bibliothèque scientifique de McGill possède une politique de promotion: «Nous nous rendons dans les classes pour annoncer les séances de formation», et le taux de participation est satisfaisant.

On remarque donc qu'une attitude active de la part du personnel peut faire la différence dans certains cas. Mais une 
politique de marketing, à elle seule, ne suffit pas à amener la clientèle à suivre des séances de formation.

\section{Statistiques sur l'utilisation des catalogues en ligne}

Seule la bibliothèque scientifique de McGill possède des statistiques sur le nombre d'utilisateurs formés (75\%). À la Bibliothèque des sciences de l'UQAM, on estime à moins de un pourcent la proportion d'usagers formés. Quant à la Bibliothèque de l'École Polytechnique, un peu moins de la moitié des étudiants nouvellement arrivés font une demande d'aide auprès des bibliothécaires (formation individuelle).

Les chiffres sont éloquents : McGill et I'UQAM constituent les deux extrêmes. Cependant, leurs ressources respectives sont aussi à l'opposé. Ainsi, le personnel de la Bibliothèque des sciences de l'UQAM se voit forcé, avec des ressources limitées, à former le plus d'étudiants possible dans une période de temps très courte. C'est pourquoi les séances de formation accueillent autant d'étudiants à la fois.

Quant aux bibliothécaires de l'École Polytechnique, ils sont obligés d'adopter une sorte de plan d'urgence qui se limite au principal problème que présente le système, à savoir l'absence d'accès par sujet. Selon nous, la non-participation du corps enseignant aux programmes de formation destinés aux étudiants de premier cycle est l'un des principaux facteurs expliquant cette situation.

Àla bibliothèque scientifique de McGill, on constate que différents moyens ont été mis en place, mais derrière tous ces moyens, on sent qu'il y a une volonté et ce, à différents paliers. Une volonté au niveau de la direction de la bibliothèque qui a certainement consenti à dégager les fonds nécessaires de manière à ce qu'il y ait des ressources matérielles et humaines suffisantes pour soutenir un programme de formation. On retrouve aussi une volonté de la part des professeurs qui semblent comprendre l'importance d'une telle formation. Enfin, soulignons le rôle actif et dynamique que joue le personnel de la bibliothèque dans le processus de la formation des catalogues en ligne. Mais le plus important, jugeons-nous, est cette volonté de penser la formation des utilisateurs en fonction des usagers euxmêmes : pensons aux trois niveaux de formation qui tentent de suivre l'étudiant tout au cours de son cheminement scolaire, et à la possibilité de formation continue (l'étudiant peut donc se présenter à la séance de formation au moment qu'il juge opportun).

On peut aussi penser qu'avec un taux de réussite semblable, une sorte de tradition est ancrée chez les étudiants, tradition favorable à l'idée de la formation documentaire tout au cours de leur cheminement scolaire.

\section{Conclusion}

Tous ces éléments, croyons-nous, sont autant de facteurs de réussite pour un programme de formation aux catalogues en ligne, qu'ils s'appellent MUSE, ARCHIMEDE ou encore, BADADUQ. On peut toujours rêver aux systèmes "d'aide invisible» tels que développés pour les catalogues en ligne de troisième génération. Nous sommes cependant de l'avis de Carol Tenopir ${ }^{11}$ qui souligne que les systèmes ne remplaceront jamais l'aide intermédiaire et qu'une aide humaine sera toujours nécessaire, surtout lorsque se présentent des demandes complexes. II s'agit encore de trouver la forme d'aide que préfèrent les usagers ${ }^{12}$, de bien comprendre et de s'adapter à leurs besoins et à leurs difficultés. Obliger l'usager à s'adapter aux types de formation ne peut que nuire aussi bien à l'usager qu'au programme mis en place. En ce sens, une volonté, au niveau gouvernemental, d'intégrer la formation documentaire à la vie scolaire et ce, dès le primaire, ne serait que souhaitable. Ainsi, peut-être arriverions-nous à éviter qu'il y ait autant de perpétuels novices et d'utilisateurs qui connaissent plus ou moins les outils documentaires mis à leur disposition.

11. Carol Tenopir, "An Interface for Self-Service Searching), Library Journal, (September 1988), 143.

12. Voirà ce sujet: Joan Cherry and Marshall Clinton, "A Profile of User...", p. 121-128. 